\title{
FLAT MANIFOLDS WITH HOMOGENEOUS HOLONOMY REPRESENTATION
}

\author{
R. LUTOWSKI
}

\begin{abstract}
We show that a rational holonomy representation of any flat manifold except torus must have at least two non-equivalent irreducible subrepresentations. As an application we show that if a Kähler flat manifold is not a torus then its holonomy representation is reducible.
\end{abstract}

\section{INTRODUCTION}

Let $n \in \mathbb{N}$. Let $\Gamma \subset \operatorname{Isom}\left(\mathbb{R}^{n}\right)$ be a crystallographic group, i.e. a discrete and cocompact subgroup if isometries of the $n$-dimensional euclidean space $\mathbb{R}^{n}$. By the first Bieberbach theorem (see [6, Theorem 2.1], [1, Theorems 3.1]) $\Gamma$ fits into the following short exact sequence

$$
0 \longrightarrow M \longrightarrow \Gamma \stackrel{\pi}{\longrightarrow} G \longrightarrow 1
$$

where $G$ is a finite group and $M$ is a maximal abelian normal subgroup of $\Gamma$. Moreover, $M$ is free abelian group of rank $n$ and it admits a structure of a faithful $G$-module, defined by the conjugations in $\Gamma$. The representation $\varphi: G \rightarrow \mathrm{GL}(M)$ which corresponds to this module is called the integral holonomy representation of $\Gamma$.

If $\Gamma$ is torsion-free we call it a Bieberbach group. In this case the orbit space $X=\mathbb{R}^{n} / \Gamma$ is a closed Riemannian manifold with sectional curvature equal to zero, a flat manifold for short. Moreover $\Gamma$, which is isomorphic to the fundamental group of $X$, determines the manifold up to affine equivalence. It is worth to notice that - up to conjugation in $\operatorname{GL}(n, \mathbb{R})-\varphi(G)$ is equal to the holonomy group of $X$.

Let $\mathbb{F}$ be a field. We will say that $G$-module $M$ is $\mathbb{F}$-homogeneous if $\mathbb{F} G$-module $\mathbb{F} \otimes_{\mathbb{Z}} M$ has only one homogeneous component, i.e. all of its irreducible submodules are isomorphic. In the particular case $\mathbb{F}=\mathbb{Q}$ we will call $M$ homogeneous. The same nomenclature applies to the corresponding representations of $G$.

In this paper we prove the following theorem:

Theorem 1. The only Bieberbach groups with homogeneous integral holonomy representation are the fundamental groups of flat tori.

2010 Mathematics Subject Classification. Primary: 20H15, Secondary: 20C20, $57 \mathrm{~S} 30$.

Key words and phrases. Bieberbach group, flat manifold, Kähler manifold, homogeneous representation, holonomy representation. 
We use the theorem to prove that the holonomy representation of any Kähler flat manifold may not be $\mathbb{C}$-homogeneous and in particular irreducible. Both of these results are generalization of the one presented in [4] where the authors consider the case of $\mathbb{Q} \otimes_{\mathbb{Z}} M$ being irreducible.

The paper is organized as follows: in Section 2 we recall a notion of lattices and sublattices. Section 3 describes a reduction of the problem of homogeneous flat manifolds to the case where the holonomy group is simple. It is in fact a generalization of [4, Section 2] to the homogeneous case. The next two sections are devoted to the proof of Theorem 1 and its application to the class of Kähler flat manifolds.

\section{LATTICES}

Remark 2.1. Since we consider tensor product over integers only, we will omit the subscript $\mathbb{Z}$ from now on.

Let $G$ be a finite group. For the convenience of our further considerations we introduce a notion of a $G$-lattice, i.e. a $G$-module which is a free $\mathbb{Z}$-module of finite rank. A $G$-submodule $M^{\prime}$ of $M$ is called a sublattice if it is pure as $\mathbb{Z}$-submodule. As a consequence of this definition, we will call $M$ an irreducible lattice if its only submodule of lower rank is 0 or, equivalently, $\mathbb{Q} \otimes M$ is a simple $\mathbb{Q} G$-module. This approach allows us to build a descending chain

$$
M=M_{k} \supset M_{k-1} \supset \ldots \supset M_{0}=0
$$

of $G$-modules such that $M_{i-1}$ is a maximal sublattice of $M_{i}$ and hence $M_{i} / M_{i-1}$ is an irreducible $G$-lattice, for $i=1, \ldots, k$. Hence we can speak about composition series and composition factors for lattices (see [2, §73] for more detailed description).

\section{REDUCTION}

As in the previous section, let $G$ be a finite group. For any $G$-lattice $M$ denote by $\operatorname{Irr}(G, M)$ the set of irreducible characters arising from constituents of $\mathbb{C} \otimes M$. Note that if $M$ is a homogeneous $G$-lattice and $S$ is a composition factor of $M$ then $\operatorname{Irr}(G, M)=\operatorname{Irr}(G, S)$. We immediately get the following generalization of [4, Lemma 2.1]:

Lemma 3.1. Let $M$ be a homogeneous $G$-lattice. Let $p$ be a prime and $\mathbb{Z}_{p}$ - the ring of $p$-adic integers. Suppose that $\mathbb{Z}_{p} \otimes M$ contains an indecomposable direct summand in the principal $\mathbb{Z}_{p} G$-block. Then every irreducible constituent of $\mathbb{C} \otimes M$ is in the principal p-block of $G$.

Let $\Gamma$ be a crystallographic group which fits into the sequence (1.1) and let $\alpha \in H^{2}(G, M)$ be the cohomology class corresponding to this extension. Then $\Gamma$ is torsion-free if and only if $\alpha$ is special, i.e. the restriction of $\alpha$ to every subgroup of $G$ of prime order is non-zero (see [1, Theorem III.2.1] for example). 
Note that in [4, Lemma 2.2] the irreducibility of a lattice was only needed to use [4, Lemma 2.1]. Hence we can generalize it to the homogeneous case:

Lemma 3.2. Let $M$ be a homogeneous G-lattice such that $H^{2}(G, M)$ contains a special element. Let $S$ denote a simple component in the socle $\operatorname{Soc}(G)$ (the product of minimal normal subgroups) of $G$. Then we have:

(a) If $\vartheta \in \operatorname{Irr}(G, M)$, then $\vartheta$ is in the principal p-block for every prime $p$ dividing $|G|$.

(b) If $\psi \in \operatorname{Irr}(S, M)$, then $\psi$ is in the principal p-block for every prime $p$ dividing $|G|$.

(c) Let $p$ be a prime dividing $|S|$ such that a Sylow p-subgroup of $S$ is cyclic. Then there is $\Theta \in \operatorname{Irr}(S, M)$ which has the following position on the oriented Brauer tree:

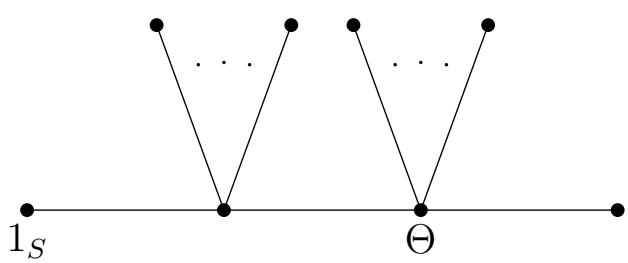

Denote by $\mathcal{S}(G)$ the set of those complex irreducible characters $\chi$ of $G$ such that for every prime $p$ dividing $|G|$ :

(1) $\chi$ is in the principal $p$-block;

(2) If Sylow $p$-subgroup of $G$ is cyclic, then $\chi$ has the position given by the position of $\Theta$ on the Brauer tree (3.1) of the principal p-block.

The base finding of [4], giving its main result, may be stated as follows:

Proposition 3.3 ([4, Section 3]). Let $S$ be a non-abelian finite simple group. Then $\mathcal{S}(S)=\emptyset$.

Since for every finite group its socle is a product of simple groups, we immediately get the following corollary.

Corollary 3.4. Let $M$ be a homogeneous $G$-lattice such that $H^{2}(G, M)$ contains a special element. Then $\operatorname{Soc}(G)$ is a product of elementary abelian groups.

\section{Proof of Theorem 1}

In the case of non $p$-groups of the proof we follow [4, Section 4].

Let $n \in \mathbb{N}, \Gamma \subset \operatorname{Isom}\left(\mathbb{R}^{n}\right)$ be a Bieberbach group which fits into the short exact sequence (1.1) and $X=\mathbb{R}^{n} / \Gamma$ be a homogeneous flat manifold, i.e. $M$ is a homogeneous $G$-lattice. Denote by $\chi$ its character. 
Assume that $G$ is a non-trivial group. Then $\operatorname{Soc}(G)$ is non-trivial. Now take any prime $p$ dividing $|\operatorname{Soc}(G)|$ and a Sylow $p$-subgroup $N$ of $\operatorname{Soc}(G)$. By Corollary $3.4 N$ is characteristic in the socle, hence it is normal in $G$.

If $q \neq p$ is another prime dividing the order of $G$ then, by Lemma 3.2 , every $\vartheta \in \operatorname{Irr}(G, M)$ is in the principal $q$-block and so, by [3, Lemma IV.4.12], has $N \subset O_{q^{\prime}}(G)$ in its kernel. But if $k$ is the composition length of $M$ then

$$
\chi=k\left(\sum_{\vartheta \in \operatorname{Irr}(G, M)} \vartheta\right)
$$

has $N$ in its kernel and $M$ is not a faithful $G$-module.

On the other hand, if $G$ is a $p$-group and $C$ is a cyclic and normal subgroup of $G$ then, by the Clifford's theorem (see [2, Theorem 49.2]), the restriction $\chi^{\prime}$ of $\chi$ to $C$ equals

$$
\chi^{\prime}=\varphi_{1}+\varphi_{2}+\ldots+\varphi_{l}
$$

where $\varphi_{1}, \ldots, \varphi_{l}$ are characters arising from irreducible components of the $\mathbb{Q} C$-module $\mathbb{Q} \otimes M$. Moreover, they are pairwise conjugated, i.e.

$$
\forall_{1 \leq i, j \leq l} \exists_{g \in G} \forall_{c \in C} \varphi_{i}(c)=\varphi_{j}\left(g c g^{-1}\right) .
$$

By [5, Theorem 7.1] if $H^{2}(C, M) \neq 0$ then there exists $1 \leq i \leq l$ s.t. $\varphi_{i}$ is the trivial character. But then the formula (4.1) shows that $\varphi_{i}$ is trivial for every $1 \leq i \leq l$. And again we get that $M$ is not a faithful $G$-module.

The above considerations show that $G$ must be the trivial group and hence $X$ is a flat $n$-dimensional torus.

\section{KÄHLER FLAT MANIFOLDS}

Let $\Gamma$ be a discrete, cocompact and torsion-free subgroup of $U(n) \ltimes$ $\mathbb{C}^{n}$. Then $X=\mathbb{C}^{n} / \Gamma$ is a Kähler flat manifold with $\pi_{1}(X) \cong \Gamma$ (see [6, Proposition 7.1]). If $\mathcal{B}: U(n) \ltimes \mathbb{C}^{n} \rightarrow O(2 n) \ltimes \mathbb{R}^{2 n}$ is a homomorphism given by

$$
\mathcal{B}(A, a)=\left(\left[\begin{array}{cc}
\operatorname{Re}(A) & -\operatorname{Im}(A) \\
\operatorname{Im}(A) & \operatorname{Re}(A)
\end{array}\right],\left[\begin{array}{l}
\operatorname{Re}(a) \\
\operatorname{Im}(a)
\end{array}\right]\right)
$$

then $\mathcal{B}(\Gamma) \subset \operatorname{Isom}\left(\mathbb{R}^{2 n}\right)$ is a Bieberbach group. Moreover if $G$ is a holonomy group and $\varphi: G \rightarrow \operatorname{GL}(n, \mathbb{C})$ is a holonomy representation of $X$ then the map $G \rightarrow \mathrm{GL}(2 n, \mathbb{R})$ defined by

$$
g \mapsto\left[\begin{array}{cc}
\operatorname{Re}(\varphi(g)) & -\operatorname{Im}(\varphi(g)) \\
\operatorname{Im}(\varphi(g)) & \operatorname{Re}(\varphi(g))
\end{array}\right]
$$

is equivalent to the (integral) holonomy representation of the flat manifold $\mathbb{R}^{2 n} / \mathcal{B}(\Gamma)$. We get 
Theorem 2. Holonomy representation of a Kähler flat manifold, which is not a flat torus, contains at least two $\mathbb{C}$-homogeneous components. In particular it is reducible.

Proof. If $\varphi$ is $\mathbb{C}$-homogeneous then the integral holonomy representation of $\mathcal{B}(\Gamma)$ is homogeneous.

\section{ACKNOWLEDGMENT}

The author would like to thank Marek Hałenda, Gerhard Hiss and Andrzej Szczepański for helpful discussions.

\section{REFERENCES}

[1] L.S. Charlap. Bieberbach groups and flat manifolds. Universitext. SpringerVerlag, New York, 1986.

[2] C.W. Curtis and I. Reiner. Representation theory of finite groups and associative algebras. Pure and Applied Mathematics, Vol. XI. Interscience Publishers, a division of John Wiley \& Sons, New York-London, 1962.

[3] Walter Feit. The representation theory of finite groups, volume 25 of NorthHolland Mathematical Library. North-Holland Publishing Co., Amsterdam-New York, 1982.

[4] Gerhard Hiss and Andrzej Szczepański. On torsion free crystallographic groups. J. Pure Appl. Algebra, 74(1):39-56, 1991.

[5] Saunders MacLane. Homology. Classics in Mathematics. Springer-Verlag Berlin Heidelberg, reprint of the 1975 edition, 1995.

[6] A. Szczepański. Geometry of crystallographic groups, volume 4 of Algebra and Discrete Mathematics. World Scientific Publishing Co. Pte. Ltd., Hackensack, NJ, 2012.

Institute of Mathematics, University of Gdańsk, Gdańsk, Poland

E-mail address: rafal.lutowski@mat.ug.edu.pl 